\title{
BIBECHANA
}

ISSN 2091-0762 (Print), 2382-5340 (Online)

Journal homepage: http://nepjol.info/index.php/BIBECHANA

Publisher: Department of Physics, Mahendra Morang A.M. Campus, TU, Biratnagar, Nepal

\section{Molecular dynamics study of structural properties of $\gamma$-aminobutyric acid (GABA)}

\author{
Shyam P. Khanal, Rajendra Prasad Koirala, Esha Mishra, Narayan P. Adhikari* \\ Central Department of Physics, Tribhuvan University, Kirtipur, Nepal \\ *Email: narayan.adhikari@cdp.tu.edu.np
}

\section{Article Information:}

Received: June 13, 2020

Accepted: June 25, 2020

Keywords:

Molecular dynamics

GABA

Radial distribution function

van der Waals radius

\begin{abstract}
The study of structural conformation of Gamma-aminobutyric acid (GABA) exhibits its biological and chemical activities. The GABA molecule is responsible in neurotransmission from one neuron to another neuron and activates the ion channels to pass the chlorine and sodium ions in nerve cells. Its conformation in solid state and gas state are extremely different and it also shows five different conformations in aqueous solution. The study of its structure in such environment can reveal its activity in cellular environment. We have performed the classical molecular dynamics study of this system of GABA in aqueous medium to deal its structure. Radial distribution function (RDF) has been used to study the structural properties of the system.
\end{abstract}

DOI: https://doi.org/10.3126/bibechana.v18i1.29442

This work is licensed under the Creative Commons CC BY-NC License. https://creativecommons.org/licenses/by-nc/4.0/

\section{Introduction}

Gamma-aminobutyric acid (GABA), a chief inhibitory neurotransmitter, plays major role in reducing the neuronal excitability throughout the central nervous system [1-3]. It is mostly found in nervous system of highly developed brain of mammals. It is, in fact, a chemical messenger that transmits the signals across chemical synapses from one neuron to another neuron, gland cells or to the muscles [4]. It is also used to treat high blood pressure, stress and anxiety; and to stimulate the secretion of natural growth hormone of body. The disorder of GABA in the body may cause the neurologic and psychiatric conditions. In addition, GABA is also detected in the other part from central nervous system like intestines, kidneys, uterus, ovaries, lungs etc. So, it has several functions in body mechanisms [5].

GABA is synthesized from anion of glutamic acid, called glutamate, via the enzyme glutamate decarboxylase with pyridoxal phosphate as a cofactor [6]. Its chemical formula is $\mathrm{C}_{4} \mathrm{H}_{9} \mathrm{NO}_{2}$ and molar mass $103.120 \mathrm{~g} / \mathrm{mol}$. It is a white microcrystalline powder with density $1.11 \mathrm{~g} / \mathrm{mL}$. It is soluble in water with solubility value $130 \mathrm{~g} / 100$ $\mathrm{mL}$. The temperature difference between solid state 
and the gas state is quite narrow, with melting point $203.7^{\circ} \mathrm{C}$ and boiling point $247.9^{\circ} \mathrm{C}[7,8]$.

It contains a primary amine group and a carboxylic acid functional group, due to which it is categorized in amino acid group; however, the amino group ($\mathrm{NH}_{2}$ ) does not link to the alpha carbon as the ordinary amino acid contains. That is why, the GABA is not incorporated into the protein molecule. It is mostly found in zwitterion form [9, 10]. The interesting characteristic of GABA is its nature of conformation with its surroundings. It is mostly found highly folded configuration in gas phase and extended form in solid phase. Its character is surprising in solvent. It has high solvent effect with five different conformation [11]. The biochemical functions of biomolecules are greatly influenced by their structural conformation. Since the GABA can be at different conformations in various phases and also in the surrounding conditions, its structural study makes the great sense to understand the neurotransmission in nervous system, the spinal cord and hyperpolarization condition in ion exchange process in nerve cells [12]. Several researches have been carried out to deal the structural conformations of GABA in aqueous solution; however its structural variations in the living body are still unclear. Solvation of GABA molecule in water resembles real body.

Ashby et al. studied about different interactions with binding site that involved in GABA binding in molecular level; and Zafar and Jabeen studied the structure and function of GABA transporters (GATs) using computational method [13, 14]. Also, many experimental studies about transport properties of GABA have been already performed. Umecky et al. and Yui et al. measured the binary diffusion coefficient of GABA in infinitesimal aqueous medium at different temperature using Taylor dispersion method $[15,16]$. Also, viscosity of aqueous solution of GABA was estimated by Romero and Beltron [17].
Molecular dynamics (MD) study can be considered as an alternative technique to study about many properties including structural analysis [18]. MD also provides guideline for experimental study. To our best knowledge, the structural properties of GABA in water have not been studied using molecular dynamics. We expect that this study will help to learn the structural conformation of the molecule in water environment.

In this paper, we have discussed methods and methodology in section 2. The results of the work are presented and discussed in section 3; and finally conclusions and concluding remarks are presented in section 4 .

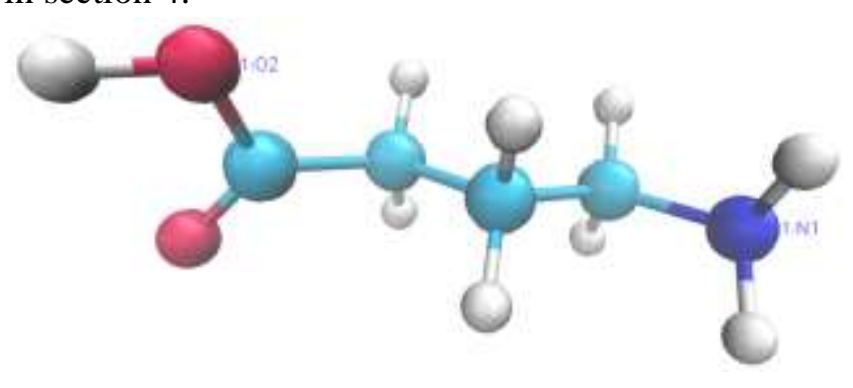

Fig. 1: Snapshot of GABA molecule.

\section{Methods and Methodology}

\section{Modeling of system}

We performed classical molecular dynamics simulations of the system of aqueous solution of GABA. GROMACS 5.1.1 software package was used for the simulations [19]. The OPLS-AA (optimized potentials for liquid simulations - all atom) force field for modeling the GABA molecule and three point SPC/E [20] water model were used during the simulations. In the classical molecular dynamics simulations, we solve the Newton's equation of motion [21]. To account the intramolecular interactions, the bonded interaction i.e. bond stretching, bond angle and dihedral potentials are considered. And, the non-bonded Coulomb and Lennard-Jones (LJ) interactions contribute to the inter-molecular interactions. The Coulomb interaction arises due to partial charges of the 
atoms in GABA and water molecules. For SPC/E water model, the partial charges of hydrogen and oxygen atoms are $+0.4238 \mathrm{e}$ and $-0.8476 \mathrm{e}$ respectively where e is elementary charge; and Lennard-Jones (LJ) parameters are $0.316 \mathrm{~nm}$ and $78.2 \mathrm{k}_{\mathrm{B}}$. Now, the inter-molecular interaction is caused due to non-bonded interactions which can be expressed as

$$
V\left(r_{i j}\right)=4 \varepsilon\left[\left(\frac{\sigma}{r}\right)^{12}-\left(\frac{\sigma}{r}\right)^{6}\right]+\frac{q_{i} q_{j}}{4 \pi \epsilon_{m} r_{i j}}
$$

where $r_{i j}$ is the distance between $i^{\text {th }} \& j^{\text {th }}$ atoms of charges $q_{i} \& q_{j}$ respectively, $\varepsilon \& \sigma$ are LJ parameters and $\epsilon_{m}$ is permittivity of medium between the charge.

\section{Computational details}

We performed the simulations of the system of $3 \gamma$ aminobutyric acid (GABA) as solute and 1035 water molecules as solvent at 1 atm pressure at five different temperature: $298.2 \mathrm{~K}, 303.2 \mathrm{~K}, 313.2 \mathrm{~K}$, 323.2 $\mathrm{K}$ and 333.2. The simulations were carried out in cubic simulation box under periodic boundary conditions (PBC). At first, to remove van der Waals bad contact and to obtain the minimum potential energy state, energy minimization of the system was carried out using Steepest-descent

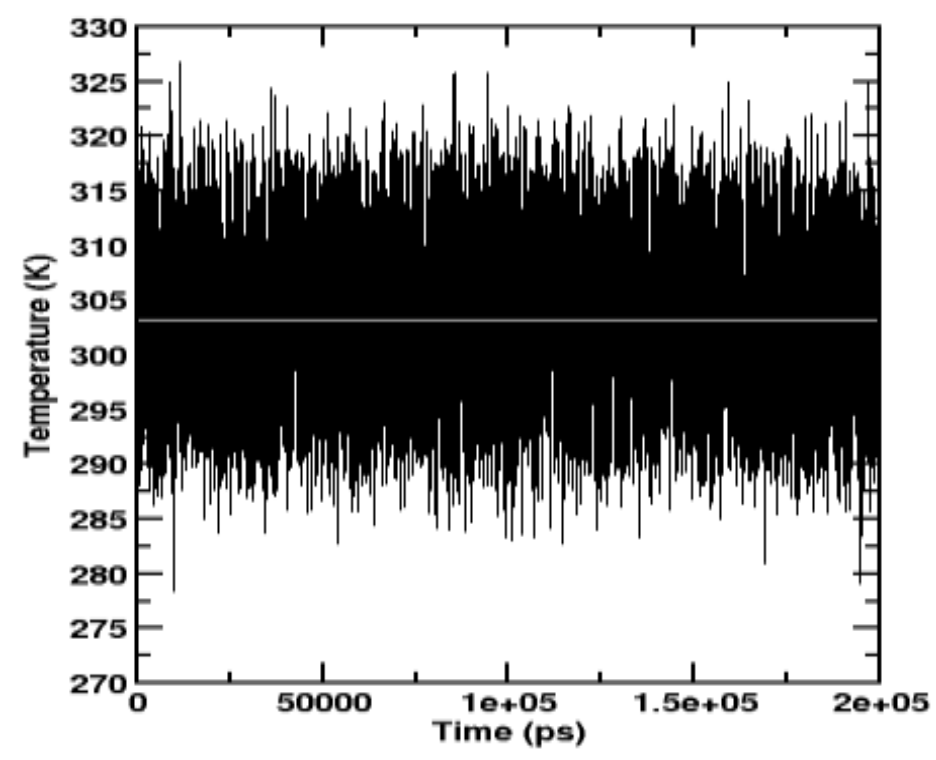

method taking $50 \mathrm{~kJ} /$ mole-nm force tolerance [22, 23].

Many properties of the system under study depend upon the parameters like temperature, pressure etc. So, the system must be in thermodynamics equilibrium. For this, the system was equilibrated at each temperature for $200 \mathrm{~ns}$ time taking time step of 0.002 ps using isothermal-isobaric (NPT) ensemble. During equilibration run, velocity rescaling thermostat with 0.01 ps coupling time was used to control temperature and Beresdsen barostat with coupling time of 0.8 ps was used to keep constant pressure [24]. LINCS algorithm and Maxwell-Boltzmann distribution were taken to constraint all bonds and to assign initial velocities for each particle respectively [24]. Also, Particle Mesh Ewald (PME) method was chosen to account the long range Coulomb interaction; and cut off parameters of $1 \mathrm{~nm}$ was taken for both short range Coulomb \& Lennard-Jones (LJ) interactions. In order to solve the equations of motion, Leap-frog algorithm was used [24]. Figure 2 represents the temperature and density profiles of the system after equilibration run at temperature of $303.2 \mathrm{~K}$. Also, the simulated values of temperature and density are presented in the table 1.

Fig. 2: Temperature (left) and density (right) profiles of the system after equilibration run at $303.2 \mathrm{~K}$ temperature. 
Table 1: Simulated values of temperature and density at five different coupling temperature.

\begin{tabular}{|c|c|c|c|}
\hline $\begin{array}{c}\text { Coupling Temperature } \\
(\mathbf{K})\end{array}$ & $\begin{array}{c}\text { Simulated } \\
\text { Temperature }(\mathbf{K})\end{array}$ & $\begin{array}{c}\text { Simulated Density } \\
\left(\mathbf{k g} / \mathbf{m}^{\mathbf{3}}\right)\end{array}$ & $\begin{array}{c}\text { Experimental Density } \\
\left(\mathbf{k g} / \mathbf{m}^{\mathbf{3}}\right)[\mathbf{2 5}]\end{array}$ \\
\hline 298.2 & $298.20 \pm 0.01$ & $990.07 \pm 0.02$ & 997.03 \\
\hline 303.2 & $303.19 \pm 0.00$ & $987.49 \pm 0.02$ & 995.63 \\
\hline 313.2 & $313.19 \pm 0.04$ & $981.46 \pm 0.03$ & 992.19 \\
\hline 323.2 & $323.19 \pm 0.01$ & $974.45 \pm 0.02$ & 988.19 \\
\hline 333.2 & $333.19 \pm 0.02$ & $967.19 \pm 0.02$ & 983.17 \\
\hline
\end{tabular}

From the table, it is seen that the simulated values of densities at different coupling temperature agree within $2 \%$ with previously reported experimental values.

After the equilibrating the system, the production run was done at each temperature using canonical (NVT) ensemble. During the production run, velocity rescaling thermostat with coupling time of $0.01 \mathrm{ps}$ was used to control temperature. The velocities of final step of equilibration run were taken as initial velocities for production run. Each production was performed for $100 \mathrm{~ns}$ with time step 0.002 ps.

\section{Results and Discussion}

In this section, we present the RDF between different atoms of GABA and Water molecules.

\section{Radial Distribution Function (RDF)}

Structural properties of the system has been studied by using radial distribution function (RDF). RDF, that provides the idea of distribution of molecules around another molecule which is taken as reference, gives the probability of finding a pair of atoms located at distance 'r' [26]. For liquid, RDF shows an oscillation up to certain distance and becomes unity which means that there is no correlation between molecules after the distance [27, 28]. The GABA molecule contains the functional groups amide $\left(-\mathrm{NH}_{2}\right)$ and carboxyl $(-\mathrm{COOH})$. Thus, to find the structural properties of the system under study, we have calculated the RDF of oxygen of water \& oxygen of water ( $\mathrm{g}$ owow $(r))$, oxygen of water \& nitrogen of amide ($\mathrm{NH}_{2}$ ) group of GABA ( $\mathrm{g}$ ow-N1 $(\mathrm{r})$ ), and oxygen of water \& oxygen of carboxylic group $(-\mathrm{COOH})$ of GABA (g ow-O2(r)). Figures 3, 4 and 5 represent the $\mathrm{g}$ ow-ow $(\mathrm{r}), \mathrm{g}$ ow-N1 $(\mathrm{r})$ and $\mathrm{g}$ ow-O2(r) at five different temperature $298.2 \mathrm{~K}, 303.2 \mathrm{~K}, 313.2 \mathrm{~K}$, $323.2 \mathrm{~K}$ and $333.2 \mathrm{~K}$ respectively.

In the RDF plots, there is a region from the reference atom up to which the value of RDF is zero. In this region, the probability of finding another atom is zero. Such region is known as excluded region (ER). Beyond the zero probability region, some peaks are observed and the value of RDF becomes unity beyond certain distance from reference atom. The unity value of RDF indicates that no correlation between atoms at that region i.e. correlation between atoms takes place up to certain distance from reference position [23]. Three peaks are observed between excluded and unity regions. The peaks indicate the favorable position of the atoms/molecules from reference. The first peak means the most favorable position of the atoms from reference. The values of excluded region (ER), first peak position (FPP), first peak value (FPV) ), second peak position (SPP), second peak value (SPV) ), third peak position (TPP) and third peak value (FPV) for $\mathrm{g}$ ow-Ow(r), $\mathrm{g}$ ow-N1 $(\mathrm{r})$ and $\mathrm{g}$ Ow-O2(r) are presented in Tables 2, 3, and 4 respectively. 


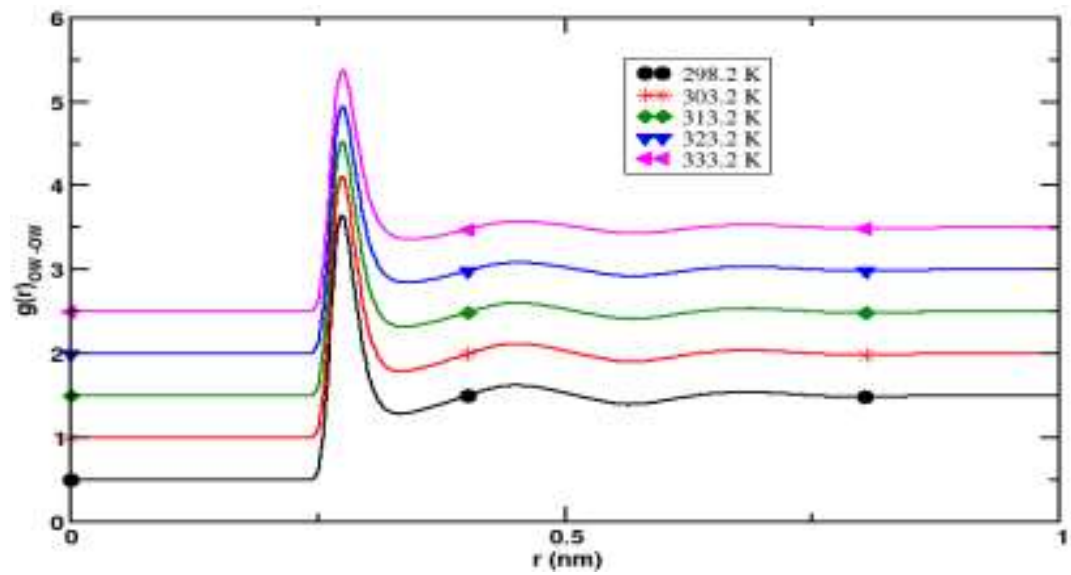

Fig. 3: RDF between oxygen atoms of water (g ow-ow(r)) at different temperature.

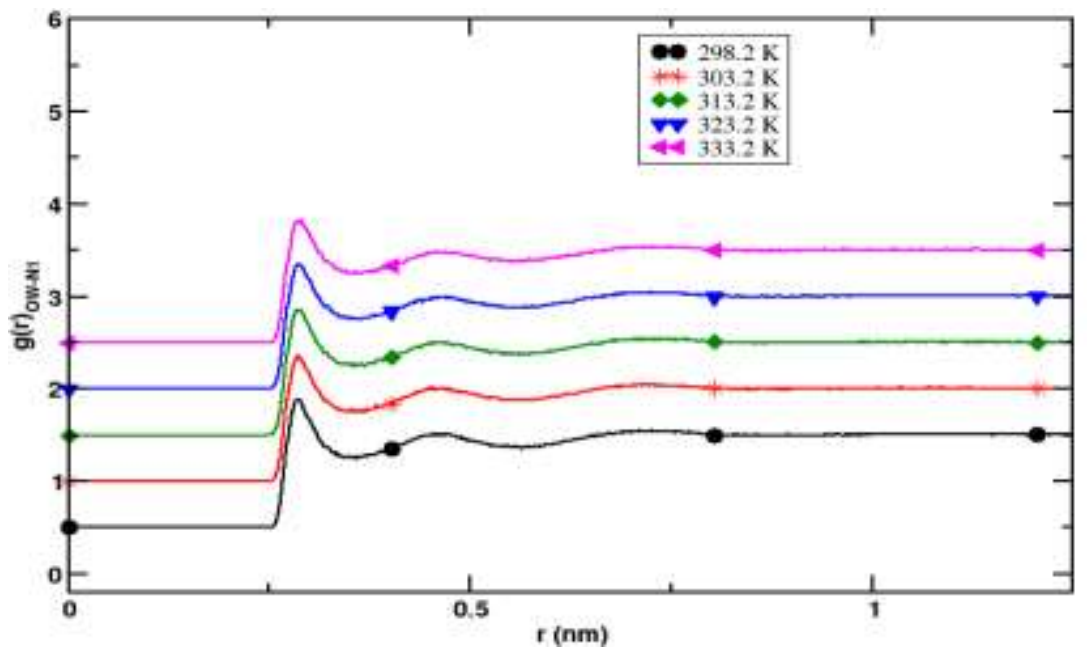

Fig. 4: RDF between nitrogen of GABA and oxygen of water $(\mathrm{g}$ ow-N1 $(\mathrm{r}))$ at different temperature.

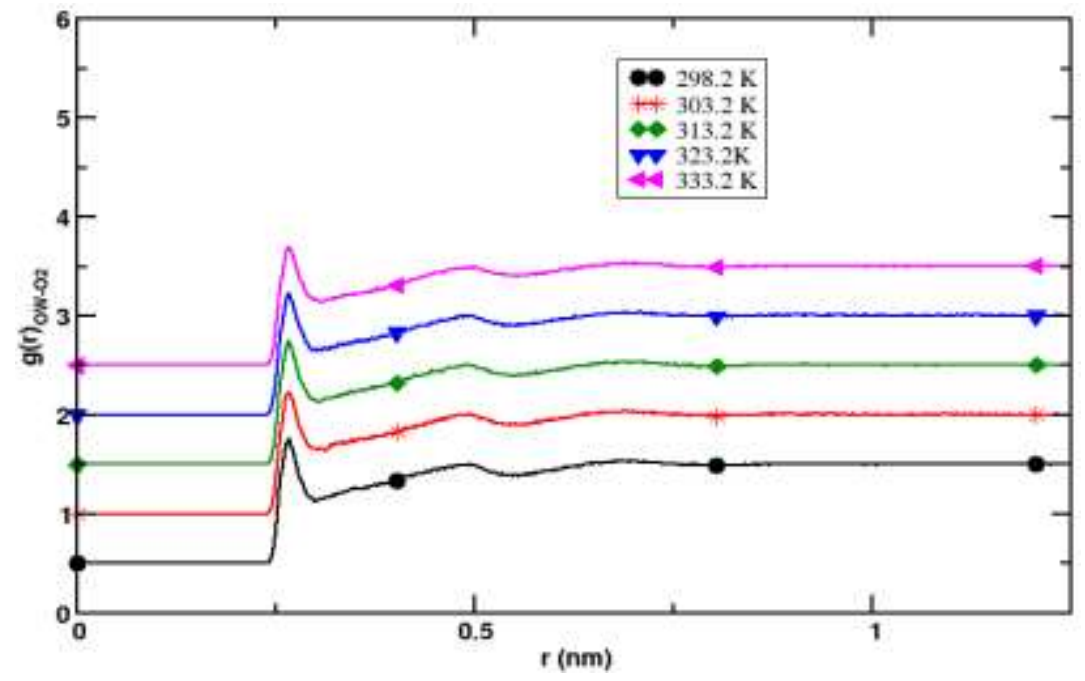

Fig. 5: RDF between oxygen of GABA and oxygen of water (g ow-o2(r)) at different temperature. 
Table 2: Simulated data for Radial distribution function (RDF) between water molecules (g ow-ow(r)) at different temperature.

\begin{tabular}{|c|l|l|l|l|l|l|l|}
\hline Temperature(K) & ER(nm) & FPP(nm) & FPV & SPP(nm) & SPV & TPP(nm) & TPV \\
\hline 298.2 & 0.240 & 0.274 & 3.141 & 0.450 & 1.126 & 0.686 & 1.046 \\
\hline 303.2 & 0.240 & 0.276 & 3.104 & 0.450 & 1.120 & 0.690 & 1.040 \\
\hline 313.2 & 0.240 & 0.276 & 3.018 & 0.450 & 1.103 & 0.688 & 1.037 \\
\hline 323.2 & 0.240 & 0.276 & 2.936 & 0.450 & 1.087 & 0.694 & 1.036 \\
\hline 333.2 & 0.240 & 0.276 & 2.874 & 0.450 & 1.075 & 0.686 & 1.034 \\
\hline
\end{tabular}

Table 3: Simulated data for Radial distribution function (RDF) between nitrogen (N1) of GABA and water molecules ( $\mathrm{g}$ ow-N1 $(\mathrm{r}))$ at different temperature.

\begin{tabular}{|c|l|l|l|l|l|l|l|}
\hline Temperature(K) & ER(nm) & FPP(nm) & FPV & SPP(nm) & SPV & TPP(nm) & TPV \\
\hline 298.2 & 0.248 & 0.284 & 1.379 & 0.466 & 1.016 & 0.726 & 1.047 \\
\hline 303.2 & 0.248 & 0.286 & 1.352 & 0.452 & 1.017 & 0.712 & 1.047 \\
\hline 313.2 & 0.246 & 0.284 & 1.359 & 0.470 & 1.004 & 0.730 & 1.048 \\
\hline 323.2 & 0.248 & 0.286 & 1.344 & 0.468 & 0.997 & 0.724 & 1.045 \\
\hline 333.2 & 0.248 & 0.290 & 1.321 & 0.458 & 0.985 & 0.726 & 1.040 \\
\hline
\end{tabular}

Table 4: Simulated data for Radial distribution function (RDF) between oxygen (O2) of GABA and water molecules (g ow-O2(r)) at different temperature.

\begin{tabular}{|l|l|l|l|l|l|l|c|}
\hline Temperature(K) & ER(nm) & FPP(nm) & FPV & SPP(nm) & SPV & TPP(nm) & TPV \\
\hline 298.2 & 0.234 & 0.266 & 1.258 & 0.494 & 1.003 & 0.700 & 1.039 \\
\hline 303.2 & 0.236 & 0.266 & 1.231 & 0.494 & 1.008 & 0.700 & 1.039 \\
\hline 313.2 & 0.234 & 0.266 & 1.242 & 0.490 & 1.006 & 0.684 & 1.034 \\
\hline 323.2 & 0.234 & 0.266 & 1.228 & 0.494 & 0.999 & 0.690 & 1.037 \\
\hline 333.2 & 0.234 & 0.266 & 1.188 & 0.488 & 0.991 & 0.684 & 1.031 \\
\hline
\end{tabular}

The values of Lennard-Jones parameter $(\sigma)$ for OW-OW, OW-N1 and OW-O2 are $0.316 \mathrm{~nm}, 0.323$ $\mathrm{nm}$ and $0.308 \mathrm{~nm}$ respectively. And, the calculated values of van der Waals radius $\left(2^{1 / 6} \sigma\right)$ for OW-OW, OW-N1 and OW-O2 are $0.355 \mathrm{~nm}, 0.363 \mathrm{~nm}$ and $0.346 \mathrm{~nm}$ respectively. From the Tables $2-4$, it is clearly observed that the values of first peak position (FPP) are smaller than the respective van der Waals radius, which indicates that other potentials along with Lennard-Jones (LJ) also contribute for stability of the system. On the other hand, Coulomb interactions arise in the system due to partial charges of hydrogen and oxygen of
SPC/E water model, and of N1 from -NH2 \& O2 from - $\mathrm{COOH}$ group of GABA molecule. Thus, both Coulomb as well as LJ including many body effects are responsible for structural properties of the system $[19,20]$. From the Table, it is also observed that the values of excluded region (ER) are less than corresponding values of the van der Waals radius.

\section{Conclusions and concluding remarks}

We performed classical molecular dynamics of a system of $3 \gamma$-aminobutyric acid (GABA) as solute and 1035 water as solvent at five different 
temperature: $298.2 \mathrm{~K}, 303.2 \mathrm{~K}, 313.2 \mathrm{~K}, 323.2 \mathrm{~K}$ and $333.2 \mathrm{~K}$ using GROMACS 5.1.1 package. During the simulations, SPC/E water model and OPLS-AA force field parameters were used. Radial distribution function (RDF) was taken to analyze the structural properties of the system. For this, we plotted the RDF between different atoms at five different temperature i.e. (i) oxygen atoms of water molecules (g ow-ow(r)), (ii) nitrogen of - $\mathrm{NH} 2$ group of GABA and oxygen of water ( $\mathrm{g}$ ow-N1 $(\mathrm{r}))$ and (iii) oxygen of - $\mathrm{COOH}$ group of GABA and oxygen of water ( $\mathrm{g}$ ow-O2 $(\mathrm{r}))$. From all the plots, we observe that both excluded region (ER) as well as first peak position (FPP) are smaller than the corresponding van der Waals radius. This indicates that along with Lennard Jones (LJ), Coulomb potential which arises due to the partial charges of different atoms as well as many body effects also contribute to the structural properties of the system.

In near future, we intend to study about the free energy calculation of the system.

\section{Acknowledgements}

SPK and RPK acknowledge the partial financial support from Nepal Academy of Science and Technology (NAST). EM acknowledges the master thesis grants support from University Grants Commission (UGC), Nepal. NPA acknowledges the UGC Award no. CRG-73/74-S\&T-01.

\section{References}

[1] H. Lodish et al. Neurotransmitters, synapses, and impulse transmission, In Molecular Cell Biology, 4th edition. WH Freeman, (2000).

[2] L, Sivilotti \& A. Nistri, = GABA receptor mechanisms in the central nervous system, Progress in Neurobiology 36(1) (1991) 35 92.

https://doi.org/10.1016/0301-0082(91)90036-Z

[3] K. Łątka, J. Jończyk, and M. Bajda, Structure modeling of $\gamma$-aminobutyric acid transportersMolecular basics of ligand selectivity, Inter. J. Biol. Macromol (2020). https://doi.org/10.1016/j.ijbiomac.2020.04.263

[4] T. A. Rocheleau, J. C. Steichen \& A. E. Chalmers, A point mutation in a Drosophila GABA receptor confers insecticide resistance, Nature 363(6428) (1993) 449-451. https://doi.org/10.1038/363449a0

[5] D. Ma et al., Structure and mechanism of a glutamate-GABA antiporter, Nature 483 (7391) (2012) 632-636. https://doi.org/10.1038/nature10917

[6] P. Rorsmanet et al., Glucose-inhibition of glucagon secretion involves activation of GABA A-receptor chloride channels, Nature 341(6239) (1989) 233236. https://doi.org/10.1038/341233a0

[7] A. J. Dobson \& R. E. Gerkin, $\gamma$-Aminobutyric acid: a novel tetragonal phase, Acta Crystallographica Section C: Crystal Structure Communications 52(12) (1996) 3075-3078.

https://doi.org/10.1107/S0108270196010001

[8] O. A. Petroff, Book review: GABA and glutamate in the human brain, The Neuroscientist 8(6) (2002) 562-573. https://doi.org/10.1177/1073858402238515

[9] A. Schousboe \& H. S. Waagepetersen, GABA: homeostatic and pharmacological aspects, Progress in Brain Research 160 (2007) 919.

https://doi.org/10.1016/S0079-6123(06)60002-2

[10] N. Ottosson, M. Pastorczak, S. T. van der Post, \& H. J. Bakker, Conformation of the neurotransmitter $\gamma$-aminobutyric acid in liquid water, Physical Chemistry Chemical Physics 16(22) (2014) 10433-10437. https://doi.org/10.1039/C4CP00671B

[11] G. Deniau, et al., Synthesis, Conformation and Biological Evaluation of the Enantiomers of 3Fluoro- $\gamma$-Aminobutyric Acid ((R)-and (S)-3FGABA): An Analogue of the Neurotransmitter GABA, Chem Bio Chem 8(18) (2007) 2265-2274. https://doi.org/10.1002/cbic.200700371

[12] C. Lee, \& A. J. de Silva, Interaction of Neuromuscular Blocking Effects of Neomycin and Polymyxin B, Anesthesiology: The Journal of the American Society of Anesthesiologists 50(3) (1979) 218-220. https://doi.org/10.1097/00000542-197903000-0001

[13] J. A. Ashby, et al., GABA binding to an insect GABA receptor: a molecular dynamics and mutagenesis study, Biophysical Journal 103(2012) 2071-2081. https://doi.org/10.1016/j.bpj.2012.10.016

[14] S. Zafar, and I.t Jabeen, Structure, function, and modulation of $\gamma$-aminobutyric acid transporter 1 (GAT1) in neurological disorders: a pharmacoinformatic prospective, Frontiers in Chemistry 6 (2018) 397. https://doi.org/10.3389/fchem.2018.00397 
[15] T. Umecky, K. Ehara, S. Omori, T. Kuga, K. Yui, and T. Funazukur, Binary diffusion coefficients of aqueous phenylalanine, tyrosine isomers, and aminobutyric acids at infinitesimal concentration and temperatures from (293.2 to 333.2$) \mathrm{K}$, Journal of Chemical \& Engineering Data 58 (2013) 19091917. https://doi.org/10.1021/je3012698

[16] K. Yui, Y. Noda, M. Koido, M. Irie, I. Watanabe, T. Umecky and T. Funazukur, Binary Diffusion Coefficients of Aqueous Straight-Chain Amino Acids at Infinitesimal Concentration and Temperatures from (298.2 to 333.2) K, Journal of Chemical \& Engineering Data 58 (2013) 28482853. https://doi.org/10.1021/je301370s

[17] C. M. Romero and A. Beltrán, Effect of temperature and concentration on the viscosity of aqueous solutions of 3-aminopropanoic acid, 4aminobutanoic..., Revista Colombiana de Química 41 (2012) 123-131.

[18] M. P. Allen and D. Tildesley, Computer Simulation of Liquids New York, USA: Oxford University Press, 1991.

[19] D. van der Spoel, et al., GROMACS: fast, flexible, and free, Inc. J Comput Chem 26 (2005) 17011718. https://doi.org/10.1002/jcc.20291

[20] H. J. C. Berendsen J. R. Grigera, and T. P. Straatsma, The missing term in effective pair potentials, Journal of Physical Chemistry 91 (1987) 6269-6271.
[21] M. E.Tuckerman, Mark, Statistical mechanics: theory and molecular simulation, Oxford university press, 2010.

[22] S. P. Khanal, Y. P. Kandel, and N. P. Adhikari, Transport properties of zwitterion glycine, diglycine, and triglycine in water, AIP Advances 9 (2019) 065303. https://doi.org/10.1063/1.5099069

[23] R. P. Koirala, et al, Effect of temperature on transport properties of cysteine in water, AIP Advances 10 (2020) 025122. https://doi.org/10.1063/1.5132777

[24] M. J. Abraham, et al., GROMACS User Manual Version 5.1. 1, GROMACS Development Team (2016).

[25] www.mrbigler.com/misc/Pvap-H2O.PDF

[26] D. A. McQuarrie, Statistical Mechanics, Harper and Row, New York 1976.

[27] S. Pokharel, N. Pantha, and N. P. Adhikari, Diffusion coefficients of nitric oxide in water: A molecular dynamics study, International Journal of Modern Physics B 30 (2016) 1650205. https://doi.org/10.1142/S0217979216502052

[28] K. Sharma and N. P. Adhikari, Temperature dependence of diffusion coefficient of nitrogen gas in water: A molecular dynamics study, International Journal of Modern Physics B 28 (2014) 1450084. https://doi.org/10.1142/S0217979214500842 